\title{
Mon cheminement politique avec Éloi Machoro (1972-1985) de François Burck
}

\section{Hamid Mokaddem}

\section{(2) OpenEdition}

1 Journals

\section{Édition électronique}

URL : http://journals.openedition.org/jso/6868

DOI : $10.4000 /$ jso.6868

ISSN : 1760-7256

\section{Éditeur}

Société des océanistes

\section{Édition imprimée}

Date de publication : 15 octobre 2013

Pagination : 254-257

ISBN : 978-2-85430-035-2

ISSN : 0300-953x

\section{Référence électronique}

Hamid Mokaddem, « Mon cheminement politique avec Éloi Machoro (1972-1985) de François Burck », Journal de la Société des Océanistes [En ligne], 136-137 | 2013, mis en ligne le 20 octobre 2013,

consulté le 23 septembre 2020. URL : http://journals.openedition.org/jso/6868 ; DOI : https://doi.org/ $10.4000 / j 50.6868$

Ce document a été généré automatiquement le 23 septembre 2020.

(c) Tous droits réservés 


\title{
Mon cheminement politique avec Éloi Machoro (1972-1985) de François Burck
}

\author{
Hamid Mokaddem
}

\section{RÉFÉRENCE}

BURCK François, 2012. Mon cheminement politique avec Éloi Machoro (1972-1985), NouvelleCalédonie, les éditions de la Province des îles Loyauté, 160 p. dont une annexe « Documents » pp. 119-160 avec doc. photogr., discours et lettres d'Éloi Machoro, 5 photos couleur ${ }^{1}$.

1 François Burck est né en 1939 dans le village minier de Thio proche de Canala. Ancien séminariste et prêtre mariste, il exerça son ministère à Nouméa et dans la région de Thio, Canala, Kouaoua jusqu'en 1975. Il demanda sa réduction à l'état laïc et commença une carrière politique. De 1977 à 1984, il a été élu à l'Assemblée territoriale. Président de l'Union calédonienne (UC) de 1988 à 1996, il la quitte pour être depuis 1999 président de la Fédération des comités de coordination pour les indépendantistes (FCCI). Son compagnon de route politique Éloi Machoro a été tué le 12 janvier 1985 par des tireurs d'élite du GIGN (Groupe d'intervention de la gendarmerie nationale). Le fait que François Burck écrive en 2012 un texte sur Éloi Machoro pourrait prêter à suspicion. Il nous semble qu'on aurait tort de préjuger de l'objectivité du témoignage de François Burck sur Éloi Machoro sous prétexte qu'il lui a survécu et qu'il est devenu un professionnel de la vie politique. On se priverait d'une lecture captivante qui nous permet de comprendre les raisons de la révolution nationaliste du peuple kanak. Nous voyons quatre raisons suffisantes pour lire ce récit documenté.

2 Avant tout, il y a le plaisir du texte. Ce livre se savoure du fait de son style. L'auteur ne recourt pas à une écriture universitaire. Il cherche à expliquer au lecteur, qui n'a pas forcément acquis les outils scolaires pour décoder une prose élaborée, les raisons de 
l'implication totale de Machoro dans le combat pour la liberté du peuple kanak. On doit universaliser, élargir et raccourcir la formule : les raisons d'un combat pour la liberté. François Burck nous le dit avec simplicité. Il a le sens de la formule. Trait particulier aux hommes enracinés dans la terre de Nouvelle-Calédonie, il sait ce que veut dire ruminer des idées avant de les exprimer en public. Les mots sont macérés. Au risque de heurter le jugement du lecteur, ces manières de dire, propres aux femmes et hommes appartenant au " Pays ", nous les retrouvons en grande partie dans le style du récit de vie de l'homme politique calédonien Jacques Lafleur, L'Assiégé. Nous n'affirmons pas que les idées politiques soient similaires. Nous disons que la manière d'intérioriser un discours se retrouve dans les écritures respectives de L'Assiégé et de Mon cheminement politique avec Éloi Machoro. L'écriture de François Burck séduit du fait qu'elle est " superficielle parce que profonde ». Sans doute, ce style provient du fait que l'auteur a côtoyé de manière proche le monde et le peuple kanak. Il mesure ce que parler veut dire. Il sait également que l'obstacle pour un peuple marginalisé par l'école coloniale se traduit par les difficultés d'accès à la lecture. N'importe qui peut lire ce livre! Nous ajouterons : N'importe qui doit lire ce livre! Il exprime des choses graves avec légèreté. La distance d'un acteur vis-à-vis d'une période de l'histoire à laquelle il a appartenu est un exercice difficile. Un acteur qui devient l'auteur de l'écriture de sa propre histoire risque de provoquer des crispations et de susciter des résistances psychologiques. Nous pensons que l'épreuve est réussie.

La deuxième raison de lire Mon cheminement politique avec Éloi Machoro est que François Burck témoigne d'un moment incontournable de l'histoire contemporaine de la Nouvelle-Calédonie : "la période dite des événements ", plus précisément le commencement des "événements ", une séquence courte et intense qui va du 18 novembre 1984, date du boycott actif du statut Lemoine, jusqu'au 12 janvier 1985, mort violente (devrait-on dire " neutralisation »?, « exécution »?, « bavure "?) d'Éloi Machoro (1946-1985) ${ }^{2}$. Il est impossible de nous masquer les yeux et ne pas reconnaître qu'Éloi Machoro fut une des figures les plus emblématiques de la révolution nationaliste du peuple kanak. Pour ne pas dire la figure emblématique de ce moment. Qui pouvait oser parler d'Éloi Machoro ? Qui était le mieux placé pour écrire ce témoignage si ce n'est François Burck? Il est l'unique rescapé du bureau politique de l' UC, complètement renouvelé suite au congrès de l'uc en 1977, par « les jeunes loups de l'uc » (expression des médias locaux) : Jean-Marie Tjibaou, Yeiwéné Yeiwéné, Éloi Machoro, Pierre Declercq et... François Burck. Tous ces leaders avaient orienté le parti autonomiste calédonien vers l'idée d'indépendance kanak convertissant et transformant ce parti en un parti politique kanak indépendantiste (pp. 35-36). Tous sont tombés ! Foudroyés et assassinés par mort violente! Qu'on nous permette la référence à Hegel sur l'Histoire! Tout se passe comme si les grands hommes ne pouvaient vivre une vie de bonheur, sacrifiant leur vie pour réaliser une œuvre qui les dépasse, la cause de leur peuple. L'histoire universelle se sert des hommes pour accomplir son action sur la scène mondiale. Le survivant du nouveau bureau politique de l'uc, suite au congrès tenu à Bourail en 1977 - dans le fief d'un autre acteur politique calédonien important, Jean-Pierre Taïeb Aïfa, alors secrétaire général de l'Uc $\mathrm{C}^{33}$ - n'est autre que François Burck. Avant 1977, François Burck avait cheminé en politique avec Éloi Machoro dans le sillage du premier maire kanak de Canala, Émile Nechero, luimême catéchiste et élève de François Luneau, prêtre mariste fondateur de l'Uicalo (Union des indigènes amis de la liberté dans l'ordre). Toutes ces trajectoires politiques furent transfigurées et épousèrent chacune à leur manière la trajectoire nationale du 
peuple kanak mobilisé pour la conquête de sa souveraineté. Par conséquent, François Burck est une des personnes les mieux placées pour témoigner des actions et des discours de son compagnon de route Éloi Machoro. Certaines des décisions d'Éloi Machoro passaient par François Burck notamment par l'entremise de lettres (voir doc. 4 et 7, pp. 129-130, 150-151).

La troisième raison est qu'Éloi Machoro, mort jeune à 39 ans, personnifie une vision ou plus précisément un point de vue émanant de la région de Canala, exprimant une voix du peuple kanak au moment où les peuples des différents pays kanak se constituaient en peuple national de Kanaky. Éloi Machoro, avec d'autres acteurs politiques des autres régions ou pays kanak, inscrivait son action pour la Kanaky - ou comme l'indique le texte de l'accord de Nouméa - de la souveraineté (à conquérir) du peuple kanak. Au point de sacrifier sa vie pour que le peuple kanak soit reconnu au niveau réel. Nous pouvons interpréter ce sacrifice au sens de mobiliser l'énergie de sa vie pour construire la souveraineté de la Kanaky. Éloi Machoro concevait l'action et la décision au niveau pragmatique et révolutionnaire. Qu'est-ce que cela veut dire? Tout simplement, exercer de manière concrète et pragmatique le pouvoir politique et, si nécessaire, l'exercer contre le pouvoir politique colonial français. Nous devons citer les propos tenus en tant que secrétaire général de l'uc lors du congrès de l'uc le 1er novembre 1984 à Touho (centre nord-est de la Nouvelle-Calédonie) (voir la photographie pp. 80-81 et doc. 6, pp. 138-149). Ce congrès tenu en même temps que celui du Palika (Parti de libération kanak) qui tenait son congrès dans la tribu de Pwei, au nord du village de Touho, allait obliger le FLNKS (Front de libération nationale kanak socialiste) à boycotter de manière active les élections organisées pour le statut proposé par Georges Lemoine. En fait, nous invitons le lecteur à lire l'ensemble du livre ; le récit poignant du témoignage de François Burck et les textes (discours, lettres) d'Éloi Machoro reproduits en annexe du livre.

5 François Burck souligne le tempérament constructeur et pragmatique d'Éloi Machoro. Dans l'imaginaire des jeunes Kanak, et pas qu'eux, Éloi Machoro incarne la figure du "Che Guevara » de Kanaky (voir les portraits peints par Ignace Wéma et l'affiche de l'Association information et soutien aux droits du peuple kanak [AISDPK], p. 79). Un travail scientifique serait encore à faire, suite à bien d'autres ouvrages, sur cette période des événements pour comprendre l'indissociabilité de l'action pragmatique et pratique. L'urgence était, selon le discours de Machoro, la survie du peuple kanak que le statut Lemoine par l'aspect négatif, la face obscure de la démocratie, voulait nier en minorant par le nombre des voix la voix du peuple kanak ${ }^{4}$ :

« [...] il y a sur le territoire un rapport de forces qui est défavorable au peuple kanak, à ses intérêts de droit, à ses intérêts qui lui viennent de son droit, de son droit naturel et de son caractère de peuple indigène. Ce rapport de force est défavorable à ceux qui luttent pour le peuple kanak et pour l'avenir du peuple kanak. Hier, à l'USTKE , je disais que le slogan de l'USTKE, "Pas d'indépendance sans socialisme et pas de socialisme sans indépendance kanak", c'était un très bon slogan mais qui n'aurait pas lieu d'être si le peuple kanak n'existait plus et, ce que Jean-Marie voulait dire tout à l'heure ${ }^{6}$, c'est qu'à l'heure actuelle, avant 1984, avant la fin de cette année, le sort du peuple kanak est en train de se jouer; il se joue par l'intermédiaire du statut Lemoine. » (Extraits du discours d'Éloi Machoro, doc. 6, pp. 138-139).

6 La vision est claire. Le propos incisif et tranchant. Aussi percutant, précis et décisif que le coup de hache théâtralisé fendant l'urne électorale dans la mairie de Canala le 18 novembre 1984 (Voir les deux photographies de Louise Takamatsu prises le 18 
novembre 1984, doc., p. 158). Entre le 1er novembre 1984 et le 12 janvier 1985, François Burck décrit la série logique des événements qui ont ponctué la marche d'Éloi Machoro (chap. 3, pp. 43-62). Cette séquence d'événements intense suppose la production d'autres enquêtes pour recueillir le maximum de témoignages et de données fournies par les archives orales et écrites (médias locaux, nationaux, internationaux, rapports de gendarmerie, etc.). Après Maurice Lenormand, l'auteur fait le parallèle entre l'assassinat de Patrice Lumumba (leader charismatique de l'ancien Congo belge) et celui d'Éloi Machoro dans l'« épilogue » (pp. 99-114). Ce livre explique pourquoi Éloi Machoro assiège le village minier de Thio : contrôler le poumon économique du territoire. Thio dont François Burck est originaire et dont il décrit avec précision les marquages coloniaux miniers et religieux (pp. 70-71 et pp. 94-95). La quatrième et dernière raison est de décrire par le récit la logique de ce qu'Éloi Machoro nommait à juste titre le droit naturel du peuple indigène. Le peuple kanak avait raison de se révolter contre un faux-semblant de démocratie. Il était dans son droit contre les mécanismes juridiques et institutionnels d'une machine technocratique et bureaucratique construite pour nier l'existence du peuple en voulant délibérément démanteler l'existence idéelle du peuple et le construire en une "communauté ethnique » intégrée à la population à administrer ${ }^{7}$. Aujourd'hui, le droit naturel du peuple kanak paraît être reconnu par l'accord de Nouméa au prix d'une réforme de la Constitution de la République. Mais il avait fallu le prix d'actions considérées comme illégales et immorales par le pouvoir colonial. Aujourd'hui, ce récit documenté nous oblige à regarder le réel en face pour mieux comprendre comment les autres communautés et le peuple kanak peuvent et doivent " vivre avec " ou " en commun " sans tomber dans les logiques crispées du communautaire. Comment résoudre une équation incontournable, composer une communauté en partageant les voix, les peuples et communautés de Kanaky/Nouvelle-Calédonie ? Nous ne disons pas que le livre de François Burck apporte des éléments de réponse. Il contribue à modifier le regard négatif sur l'autre (ou l'Autre) en nous rappelant dans quelle mesure il était nécessaire pour Éloi Machoro d'agir comme il l'a fait.

\section{BIBLIOGRAPHIE}

ARENDT Hannah, 1995. Qu'est-ce que la politique ?, trad. et préface de Sylvie Courtine-Denamy, Paris, Le Seuil.

GoGNY Béalo, 2007. Thewe men Jila. La monnaie kanak en Nouvelle-Calédonie, Nouvelle-Calédonie, les éditions de la province Nord.

LAFLEUR Jacques, 2000. L'Assiégé. 25 ans de vie politique, une histoire partagée avec la Nouvelle-Calédonie, Paris, Plon.

MоKADDEM Hamid, 2011. Le discours politique kanak (Jean-Marie Tjibaou, Roch Déo Pidjot, Éloi Machoro, Raphaël Pidjot), Koohné, Nouvelle-Calédonie, les éditions de la province Nord. 


\section{NOTES}

2. ndlr. - Et de Marcel Nonnaro, son compagnon de lutte, « abattu » en même temps qu'Éloi.

3. Jean-Pierre Taïeb Aïfa, descendant des transportés algériens du xix ${ }^{\mathrm{e}}$ siècle, est l'actuel maire de Bourail. Il démissionna de son poste secrétaire général de l'uc en juillet 1977 puis il fonda en 1979 un parti centriste, la fnsc (Fédération pour une nouvelle société calédonienne).

4. Nous nous permettons de renvoyer le lecteur sur ce point précis à notre livre (Mokaddem, 2011).

5. La veille, à Nouméa, chef-lieu du territoire, Éloi Machoro avait participé à la réunion du syndicat, Union syndicale des travailleurs et des Kanak exploités, créé en 1982 et présidé par Louis Kotra Uregei, à son tour invité et présent au congrès de l'uc à Touho.

6. Jean-Marie Tjibaou venait de prononcer un discours d'ouverture pour exhorter les congressistes à boycotter le statut politique proposé par Georges Lemoine et empêcher de manière active selon les régions kanak l'organisation des élections territoriales devant élire les élus de l'Assemblée territoriale.

7. Hannah Arendt exprime une idée difficile sur la pluralité humaine et sur la technique moderne de destruction de peuple caractérisant selon elle la politique $\mathrm{du} \mathrm{xx}^{\mathrm{e}}$ siècle : «[...] le meurtre [...] d'un peuple et de sa constitution politique, lesquels sont potentiellement, et dans le cas de la constitution, conformément à l'intention qui l'a établie, immortels. Ce qu'on tue là ce n'est pas quelque chose de potentiellement immortel [...]. Lorsqu'un peuple perd sa liberté étatique, il perd sa réalité politique, quand bien même parvient-il à survivre physiquement. » (1995: 134).

1. Dont le drapeau du pays kanak créé en 1983 et la reproduction d'une peinture de Miriam Schwam, La hache d'Éloi Machoro (2011), représentant le tamioc d'Éloi Machoro lors du barrage dressé le 24 septembre 1981 à Fonhwary (proche de La Foa) contemporain de l'assassinat de Pierre Declercq, secrétaire général de l'Union calédonienne (uc). La photographie de couverture reproduit une toile de David Diaiké, peintre kanak de Canala, Généalogie d'une cérémonie coutumière kanak (1994). 